\title{
Optimisation de l'efficacité des graines de moringa oleifera dans le traitement des eaux de consommation en Afrique sub-saharienne
}

\author{
Cas des eaux du Burkina Faso
}

Optimization of the effectiveness of the Moringa oleifera seeds in the treatment of drinking water in sub-Saharan Africa Case study of the waters of Burkina Faso

\author{
Aminata Kabore, Boubacar Savadago, Francis Rosillon, Alfred S. Straore et \\ Dayéri Dianou
}

Volume 26, numéro 3, 2013

Reçu le 28 avril 2012, accepté le 21 juin 2013

URI : https://id.erudit.org/iderudit/1018786ar

DOI : https://doi.org/10.7202/1018786ar

Aller au sommaire du numéro

Éditeur(s)

Université du Québec - INRS-Eau, Terre et Environnement (INRS-ETE)

ISSN

1718-8598 (numérique)

Découvrir la revue

Citer cet article

Kabore, A., Savadago, B., Rosillon, F., Straore, A. S. \& Dianou, D. (2013). Optimisation de l'efficacité des graines de moringa oleifera dans le traitement des eaux de consommation en Afrique sub-saharienne : cas des eaux du Burkina Faso. Revue des sciences de l'eau / Journal of Water Science, 26(3), 209-220. https://doi.org/10.7202/1018786ar
Résumé de l'article

L'accès à l'eau potable demeure une très grande préoccupation en Afrique sub-saharienne, particulièrement en milieu rural où les populations sont confrontées à la gestion optimale des points d'eau, l'insuffisance d'hygiène et d'assainissement et au manque de méthodes appropriées de désinfection à l'échelle familiale. De ce fait, l'intégration de procédés biologiques de traitement des eaux de consommation pourrait être une alternative durable dans l'amélioration de la qualité des eaux de boisson, du fait de la disponibilité et de la non-toxicité des substances. La présente étude porte sur l'optimisation de l'efficacité des graines de Moringa oleifera dans l'assainissement des eaux de consommation en milieu rural au Burkina Faso. Des eaux de surface et de puits alimentant les populations ont été traitées avec différentes concentrations de coagulant de graines de $M$. oleifera. Le suivi de paramètres physico-chimiques et microbiologiques à différents temps de décantation a permis de déterminer les conditions optimales et d'évaluer l'efficacité des traitements. Des résultats, il est ressorti qu'en fonction des caractéristiques des eaux, les temps de décantation compris entre 1,5-2,0 h sont apparus suffisants pour éliminer la presque totalité des matières en suspension et des colloïdes. L'abattement de la turbidité obtenu était compris entre $24-96 \%$ pour un temps de décantation de $2 \mathrm{~h}$ et fonction de la turbidité initiale de l'eau. Pour le même temps de décantation, l'abattement microbien était de l'ordre de 82-94\% pour les coliformes fécaux, $81-100 \%$ pour Escherichia coli, $94-100 \%$ pour les streptocoques fécaux et $100 \%$ pour les kystes de Giardia intestinalis. Les traitements ont également permis de réduire les teneurs en nitrates, calcium, magnésium ainsi que la dureté totale. Par contre, une hausse des concentrations de sulfates et de matière organique a été enregistrée avec ces traitements. Ces résultats indiquent qu'en fonction des caractéristiques initiales, le traitement des eaux brutes avec les graines de M. oleifera améliore considérablement la qualité des eaux de boisson.
Ce document est protégé par la loi sur le droit d'auteur. L’utilisation des services d’Érudit (y compris la reproduction) est assujettie à sa politique d'utilisation que vous pouvez consulter en ligne.

https://apropos.erudit.org/fr/usagers/politique-dutilisation/ 


\section{OPTIMISATION DE L'EFFICACITÉ DES GRAINES DE MORINGA OLEIFERA DANS LE TRAITEMENT DES EAUX DE CONSOMMATION EN AFRIQUE SUB-SAHARIENNE : CAS DES EAUX DU BURKINA FASO}

Optimization of the effectiveness of the Moringa oleifera seeds in the treatment of drinking water in sub-Sabaran Africa: Case study of the waters of Burkina Faso

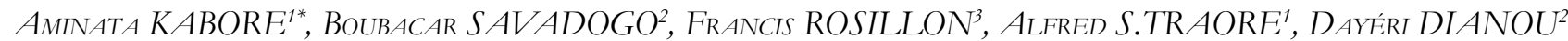

${ }^{1}$ Université de Ouagadougou, Centre de Recherche en Sciences Biologiques Alimentaires et Nutritionnelles (CRSBAN),

03 BP 7021, Ouagadougou, Burkina Faso.

${ }^{2}$ Institut de Recherche en Sciences de la Santé, Centre National de Recherche Scientifique et Technologique, 03 BP 7192, Ouagadougou, Burkina Faso.

${ }^{3}$ Université de Liège, Campus d'Arlon, Unité Eau, Environnement, Développement, 185, Avenue de Longwy, 6700 Arlon, Belgique.

Reçu le 28 avril 2012, accepté le 21 juin 2013

\section{RÉSUMÉ}

L'accès à l'eau potable demeure une très grande préoccupation en Afrique sub-saharienne, particulièrement en milieu rural où les populations sont confrontées à la gestion optimale des points d'eau, l'insuffisance d'hygiène et d'assainissement et au manque de méthodes appropriées de désinfection à l'échelle familiale. De ce fait, l'intégration de procédés biologiques de traitement des eaux de consommation pourrait être une alternative durable dans l'amélioration de la qualité des eaux de boisson, du fait de la disponibilité et de la non-toxicité des substances. La présente étude porte sur l'optimisation de l'efficacité des graines de Moringa oleifera dans l'assainissement des eaux de consommation en milieu rural au Burkina Faso. Des eaux de surface et de puits alimentant les populations ont été traitées avec différentes concentrations de coagulant de graines de $M$. oleifera. Le suivi de paramètres physico-chimiques et microbiologiques à différents temps de décantation a permis de déterminer les conditions optimales et d'évaluer l'efficacité des traitements.
Des résultats, il est ressorti qu'en fonction des caractéristiques des eaux, les temps de décantation compris entre 1,5-2,0 h sont apparus suffisants pour éliminer la presque totalité des matières en suspension et des colloïdes. L'abattement de la turbidité obtenu était compris entre 24-96\% pour un temps de décantation de $2 \mathrm{~h}$ et fonction de la turbidité initiale de l'eau. Pour le même temps de décantation, l'abattement microbien était de l'ordre de $82-94 \%$ pour les coliformes fécaux, 81-100 \% pour Escherichia coli, 94-100\% pour les streptocoques fécaux et $100 \%$ pour les kystes de Giardia intestinalis. Les traitements ont également permis de réduire les teneurs en nitrates, calcium, magnésium ainsi que la dureté totale. Par contre, une hausse des concentrations de sulfates et de matière organique a été enregistrée avec ces traitements. Ces résultats indiquent qu'en fonction des caractéristiques initiales, le traitement des eaux brutes avec les graines de $M$. oleifera améliore considérablement la qualité des eaux de boisson.

Mots clés : Eau de consommation, assainissement, Moringa oleifera, turbidité, bactéries, parasites. 


\section{ABSTRACT}

Access to safe water remains a major concern in subSaharan Africa, particularly in rural areas where populations are faced with the optimal management of water distribution points, inadequate hygiene and sanitation and a lack of appropriate methods of disinfection at the family level. Thus the integration of biological processes for the treatment of drinking water is emerging as a viable alternative for improving the quality of drinking water, because of the availability and non toxicity of the substances. This study aimed to optimize the efficiency of Moringa oleifera seeds in producing clean water for consumption in rural Burkina Faso. Surface and well water samples consumed by populations were treated with different concentrations of $M$. oleifera seeds as a coagulant. The measurement of physico-chemical and microbiological parameters, after different settling times, was used to determine the optimum conditions and assess treatment efficacy. Settling times between $90 \mathrm{~min}$ and $2 \mathrm{~h}$ appeared sufficient to remove almost all suspended solids and colloids. The reduction of turbidity obtained for a settling time of $2 \mathrm{~h}$ was 24 to $96 \%$, depending to the initial turbidity of the water samples. For the same settling time, the microbial reduction was related to the nature of the water samples and was in the range of $82-94 \%$ for fecal coliforms, $81-100 \%$ for Escherichia coli and $94-100 \%$ for faecal streptococci. By this treatment we also obtained the total elimination of cysts of Giardia intestinalis. In the treated water we also found lower levels of nitrates, calcium, magnesium and total hardness, but rising concentrations of sulfates and organic matter. These results indicate that treatment of raw water with $M$. oleifera seeds is a function of the initial quality of the water and may be sufficient to obtain clean water for human consumption.

\section{Keywords: Drinking water, sanitation, Moringa oleifera, turbidity, bacteria, parasites.}

\section{INTRODUCTION}

L'eau constitue l'une des ressources naturelles les plus importantes pour la survie de l'humanité et le développement socio-économique des nations. Aliment à part entière, toute eau destinée à la consommation humaine doit être disponible continuellement, en quantité suffisante, et ne doit pas présenter de risques pour la santé. Selon l'OMS et l'UNICEF (2000), 1,1 milliard de personnes n'ont pas accès à une eau de boisson saine. Des estimations plus récentes (WHO, 2005) indiquent que cette situation entraîne chaque année la mort de 1,6 million d'enfants par maladies diarrhéiques et près de 4 millions d'êtres humains par des maladies liées à l'eau et à l'environnement. D'après BUISSON (2001), les diarrhées comptent parmi les maladies les plus fréquentes et les plus répandues dans le monde et constituent la première cause de mortalité infantile dans les pays en développement. En Afrique sub-saharienne, les diarrhées infantiles constituent un problème de santé publique, en raison de la prolifération des germes entéropathogènes transmis le plus souvent par l'eau de boisson non traitée.

Selon le PROGRAMME DES NATIONS UNIES POUR LE DÉVELOPPEMENT (2010), l'accès à l'eau potable au Burkina Faso s'est nettement amélioré ces dernières années avec un taux national d'accès qui est passé de $18,3 \%$ en 1993 pour atteindre $66,3 \%$ en 2007. Les Nations Unies (2010) estiment que ces bons résultats ont permis au pays d'atteindre les Objectifs du Millénaire pour le Développement (OMD) dans ce secteur.

Des études récentes (AOUBA, 2012), ont montré que la situation n'est pas pour autant satisfaisante malgré les efforts faits par les gouvernements, notamment en milieu rural où les populations sont confrontées à la gestion optimale des points d'eau. Selon DIANOU et al. (2003, 2004, 2011), MAHRH (2011), PN-AEPA (2006), ROSILLON et al. (2012b) et TRAORE (2003), la qualité de l'eau consommée par les populations dans certaines zones rurales du Burkina Faso, au-delà de l'aspect quantitatif, est très préoccupante du fait de la concurrence de points d'eau traditionnels, du manque d'entretien des ouvrages hydrauliques, de l'insuffisance de l'hygiène et de l'assainissement et du manque de méthodes appropriées de désinfection à l'échelle familiale. Toujours d'après DIANOU et al. (2004, 2011), KOUKOUNARI et al. (2007) et TRAORE (2003), certaines eaux de rivière et de marigot continuent d'être utilisées en milieu rural pour la consommation humaine, notamment dans la vallée du Sourou. Il ressort clairement la nécessité de traiter les eaux de consommation dans certaines régions rurales pour un accès effectif à l'eau potable et pour l'atteinte des OMD dans ce secteur.

La coagulation-floculation est une méthode permettant d'éliminer la turbidité constituée, d'une part, de matières en suspension (minérales et organiques) ainsi que de matières organiques dissoutes et, d'autre part, de microorganismes pathogènes (bactéries, parasites et virus) (DEGREMONT 2005; DESJARDINS 1997). Ce procédé très important constitue la première étape du traitement conventionnel des eaux et utilise généralement le sulfate d'aluminium comme coagulant. Outre ces substances chimiques, des études ont montré l'efficacité de certaines substances organiques en tant que coagulant. Au regard de l'état de pauvreté des populations rurales au Burkina Faso, les méthodes viables pour le traitement des eaux, outre leur performance, doivent être simples, accessibles et de moindre coût. D'après FOLKARD et SUTHERLAND (2002), les technologies associées au traitement de l'eau doivent être aussi simples que possible, 
robustes et abordables à installer et à entretenir dans les pays en développement. Ainsi, plusieurs études (FABY et ELELI, 1993; JAHN, 1988a; KABORE, 2011) ont montré l'efficacité des graines de Moringa oleifera dans la clarification des eaux et l'élimination des microorganismes pathogènes.

La présente étude vise à mieux documenter l'influence des concentrations de Moringa oleifera et du temps de décantation pour un traitement optimum en fonction de différentes caractéristiques physico-chimiques et microbiologiques des eaux de consommation et à évaluer, en fonction des concentrations de Moringa oleifera, l'abattement des bactéries et des parasites intestinaux. L'étude vise aussi à évaluer l'effet du traitement sur certains paramètres physico-chimiques d'intérêt sanitaire, notamment les nitrates et nitrites ainsi que sur la qualité organoleptique des eaux.

\section{MATÉRIEL ET MÉTHODES}

Pour cette étude, les propriétés floculantes des graines de Moringa oleifera ont été exploitées pour réaliser la coagulation-floculation pour la clarification et l'élimination des microorganismes des eaux. La démarche a consisté à appliquer différents traitements à base de coagulant de graines de $M$. oleifera à des échantillons d'eau brute de différentes provenances du Burkina Faso utilisée pour la consommation humaine, puis à suivre leurs caractéristiques physico-chimiques et microbiologiques. L'efficacité des traitements a été évaluée en comparant les caractéristiques des échantillons traités à celles des échantillons non traités et aux normes de référence de l'OMS pour les eaux de consommation.

\subsection{Sites d'étude et échantillonnage}

Quatre sites, dont 75 échantillons d'eau provenant des barrages $\mathrm{N}^{\circ} 3$ de la ville de Ouagadougou (Ouaga 3) et de Loumbila (périphérie de Ouagadougou) dans la province du Kadiogo et utilisés pour l'approvisionnement en eau potable de la ville de Ouagadougou après traitement ainsi que ceux de la rivière Gana et du puits busé à grand diamètre de Bouaré dans la province du Sourou ont été retenus pour cette étude. Sur chaque site, les échantillons d'eau ont été collectés en triplicata dans des flacons stériles puis conservés à $4^{\circ} \mathrm{C}$ dans des glacières et rapidement transportés au laboratoire pour les traitements et analyses.

\subsection{Préparation du coagulant de Moringa oleifera}

Les graines mûres et sèches de M.oleifera obtenues au Centre National de Semences Forestières (CNSF) ont été décortiquées puis broyées selon la technique décrite par FOLKARD et SUTHERLAND (2002). La poudre fine obtenue a été utilisée pour la préparation de la solution mère de coagulant de $M$. oleifera. Pour cela, $20 \mathrm{~g}$ de poudre de graines ont été dilués dans 1 litre d'eau distillée stérilisée et le mélange agité durant une heure afin d'extraire le coagulant qui a été utilisé pour traiter les eaux.

\subsection{Traitement des échantillons d'eaux}

Au laboratoire, les échantillons ont été traités en triplicata avec des concentrations croissantes de coagulant de graines de Moringa oleifera afin de pouvoir déterminer les doses adéquates en fonction des caractéristiques des différents échantillons. Pour réaliser les jar-tests, $500 \mathrm{~mL}$ de chaque échantillon d'eau ont été introduits dans des béchers d'un floculateur à commande électrique à six postes (FC6S Jar-Test Velp Scientifica) suivi de l'ajout de différents volumes de coagulant. L'agitation des eaux après introduction du coagulant s'est faite en deux phases : une agitation rapide à 150 tours $\mathrm{min}^{-1}$ pendant cinq minutes et une agitation lente à 45 tours $\bullet \mathrm{min}^{-1}$ pendant 15 minutes.

\subsection{Caractéristiques physico-chimiques du coagulant et des échantillons d'eau}

Les caractéristiques physico-chimiques des eaux brutes ainsi que celles du coagulant extrait des graines de $M$. oleifera ont été déterminées. Les paramètres physico-chimiques de potabilité des eaux de boisson ciblés ont été la turbidité, le $\mathrm{pH}$, les concentrations en nitrates, nitrites, calcium, magnésium, sulfates, la dureté totale, la matière organique et les matières en suspension. La turbidité, le $\mathrm{pH}$ et la température ont été mesurés avant et toutes les 30 minutes durant deux heures, puis 24 heures après traitement pour tous les échantillons afin de déterminer les conditions optimales et les facteurs influençant le traitement. Les autres paramètres ont été déterminés aux conditions optimales afin d'évaluer l'effet des traitements sur la composition physico-chimiques des eaux.

La turbidité a été mesurée à l'aide d'un turbidimètre de laboratoire (550 IR WTW); le pH et la température avec un pH-mètre couplé à un thermomètre (330i WTW; pH électrode Sen Tix 41).

La matière organique a été déterminée par la méthode de perte au feu de la matière sèche. Pour cela, $100 \mathrm{~mL}$ d'eau ont été filtrés et calciné à $550{ }^{\circ} \mathrm{C}$ pendant deux heures dans un four de marque Optima selon le principe de la norme française NF 90-105. Les concentrations en calcium, magnésium, et dureté totale ont été déterminées selon les méthodes titrimétriques dont les principes sont conformes aux normes françaises, respectivement NF T 90-016 pour le calcium-magnésium, et NF T 90-003 pour la dureté totale. 
Les dosages des concentrations en nitrates, nitrites et sulfates ont été réalisés par la méthode de spectrophotométrie avec le spectrophotomètre Lange Hach DR 3800, selon les méthodes 8051, 8039, 8507 respectivement pour les sulfates, nitrates et nitrites du manuel des spectrophotomètres DR (2000,2010, $2500,2800,3800)$.

\subsection{Caractéristiques microbiologiques des eaux}

Les indicateurs microbiologiques de pollution fécale des eaux (Escherichia coli, Coliformes fécaux, Streptocoques fécaux et kystes de parasites) ont été déterminés sur tous les échantillons avant et aux conditions optimales de traitement. Les coliformes fécaux, $E$. coli et streptocoques fécaux ont été déterminés suivant la méthode de filtration sur membrane et étalement sur les milieux de culture spécifiques. Le milieu RapidE.coli pour E. coli et les coliformes fécaux et le milieu bile esculine pour les streptocoques fécaux. L'incubation a été faite à $44,5^{\circ} \mathrm{C}$ pendant $24-48$ heures dans une étuve thermostatée au laboratoire. Les kystes de parasites ont été concentrés et dénombrés au microscope suivant la méthode recommandée par WHO (2004).

Les analyses ont été effectuées dans les deux jours qui ont suivi le prélèvement, respectivement au laboratoire de l'Office National de l'Eau et de l'Assainissement pour les paramètres physico-chimiques, et au laboratoire de l'Institut de Recherche en Sciences de la Santé pour les paramètres microbiologiques.

\subsection{Analyse statistique}

Tous les résultats obtenus ont été soumis à une analyse de variance (ANOVA) avec le logiciel statistique XLSTAT-Pro 7.5 et les moyennes ont été comparées en utilisant le test de Newman Keuls au seuil de probabilité $\mathrm{p}=5 \%$.

Les paramètres mesurés ont été évalués suivant les normes WHO (2011) pour la qualité des eaux de boisson.

\section{RÉSULTATS ET DISCUSSION}

\subsection{Caractéristiques physico-chimiques des eaux et du coagulant}

Le tableau 1 montre les résultats des paramètres physicochimiques des eaux brutes et du coagulant utilisé pour le traitement. Il en ressort que les graines de $M$. oleifera sont riches en minéraux, notamment les nitrates et les sulfates, et en matière organique. Cela pourrait entraîner une augmentation de la concentration de ces minéraux et de la matière organique dans les eaux traitées. En ce qui concerne l'échantillon du Gana, les valeurs élevées des paramètres physico-chimiques sont dues aux multiples usages et à la forte activité humaine dont le site fait l'objet. En effet, le site est une rivière où l'eau est utilisée pour les activités domestiques, récréatives ainsi que pour la consommation humaine et animale. Cela explique la turbidité élevée et les fortes concentrations en matières organiques et en certains minéraux.

\section{2 Évolution de la turbidité des eaux en fonction du temps de décantation pour différentes concentrations de coagulant de M. oleifera}

Bien que la turbidité ne soit pas un indicateur direct de risque pour la santé, BRATBY (2006) a montré une forte relation entre l'abattement de la turbidité et celui des microorganismes. Aussi, la turbidité peut fournir de la nourriture et un abri aux agents pathogènes favorisant ainsi leur prolifération et des flambées de maladies d'origine hydrique (ALTAHER et ALGHAMDI, 2011), d'où l'importance de ce paramètre dans le traitement et la stabilité des eaux. La figure 1 présente l'évolution de la turbidité des eaux en fonction du temps de décantation et des concentrations de $M$. oleifera pour les différentes eaux.

De ces figures, il ressort que la clarification des eaux est rapide durant les trente premières minutes et lente après une une heure de décantation pour les différentes concentrations de $M$. oleifera. Cela s'observe pour tous les échantillons d'eau exceptés ceux de Ouaga 3 et Bouaré.

Pour les eaux brutes de Ouaga 3 qui présentaient une faible turbidité initiale (9,06 NTU) due à l'absence de colloïdes et de matières en suspension, l'introduction du coagulant a entraîné une augmentation de la turbidité des eaux suivie d'une décantation lente des particules (Figure 1b). $M$. oleifera est un coagulant primaire qui provoque la formation de ponts entre les colloïdes. Du fait de la faible présence de très peu de colloïdes dans l'eau brute, la matière organique du coagulant de $M$. oleifera entraine une augmentation de la turbidité. La turbidité des eaux après traitement est restée élevée par rapport à la turbidité initiale durant une heure et décroît pour atteindre des valeurs de l'ordre de 2 NTU au bout de 24 heures pour toutes les concentrations de $M$. oleifera, valeurs de turbidité conformes à la norme OMS pour l'eau de boisson. L'abattement de la turbidité observé a été de $24,69 \%$ pour deux heures de décantation et $76,34 \%$ pour 24 heures avec $300 \mathrm{mg} \bullet \mathrm{L}^{-1}$ de coagulant. Pour cet échantillon, les concentrations de coagulant utilisées $\left(100-300 \mathrm{mg} \bullet \mathrm{L}^{-1}\right)$ sont assez élevées, au regard de la faible turbidité initiale de l'échantillon. Les eaux de faible turbidité nécessitent des doses assez élevées car la probabilité de collisions des particules est faible (FABY et ELELI, 1993). Le traitement classique de coagulation-floculation et sédimentation n'est pas très efficace 
Tableau 1. Caractéristiques physico-chimiques du coagulant et des eaux brutes des différents sites.

Table 1. Physico-chemical characteristics of coagulant and raw water of different sites.

\begin{tabular}{|c|c|c|c|c|c|c|c|c|c|c|}
\hline & $\begin{array}{l}\text { Turbidité } \\
\text { (NTU) }\end{array}$ & $\mathrm{pH}$ & $\begin{array}{l}\text { Nitrate } \\
\left(\mathrm{mg} \bullet \mathrm{L}^{-1}\right)\end{array}$ & $\begin{array}{l}\text { Nitrite } \\
\left(\mathrm{mg} \bullet \mathrm{L}^{-1}\right)\end{array}$ & $\begin{array}{l}\text { Calcium } \\
\left(\mathrm{mg} \bullet \mathrm{L}^{-1}\right)\end{array}$ & $\begin{array}{c}\text { Magnésium } \\
\left(\mathrm{mg} \bullet \mathrm{L}^{-1}\right)\end{array}$ & $\begin{array}{l}\text { Sulfate } \\
\left(\mathrm{mg} \bullet \mathrm{L}^{-1}\right)\end{array}$ & $\begin{array}{l}\text { Dureté } \\
\text { totale }\end{array}$ & $\begin{array}{c}\mathrm{MO} \\
\left(\mathrm{mg} \bullet \mathrm{L}^{-1}\right)\end{array}$ & $\begin{array}{c}\text { MES } \\
\left(\mathrm{mg} \cdot \mathrm{L}^{-1}\right)\end{array}$ \\
\hline Loumbila & 76 & 7,7 & 1 & 0 & 4 & 12 & 9 & 16 & 12 & 39 \\
\hline Ouaga3 & 9 & 8 & 0 & 0 & 31 & 14 & 10 & 45 & 6 & 12 \\
\hline Bouaré & 150 & 8 & 10 & 0 & 39 & 17 & 12 & 46 & 38 & 116 \\
\hline Gana & 6682 & 8 & 201 & 0 & 323 & 586 & 0 & 913 & 1800 & 2917 \\
\hline $\begin{array}{l}\text { Moringa } \\
\text { oleifera }\end{array}$ & ND & ND & 34 & 4 & 0 & 0 & 47 & 0 & 14000 & ND \\
\hline
\end{tabular}

(a)

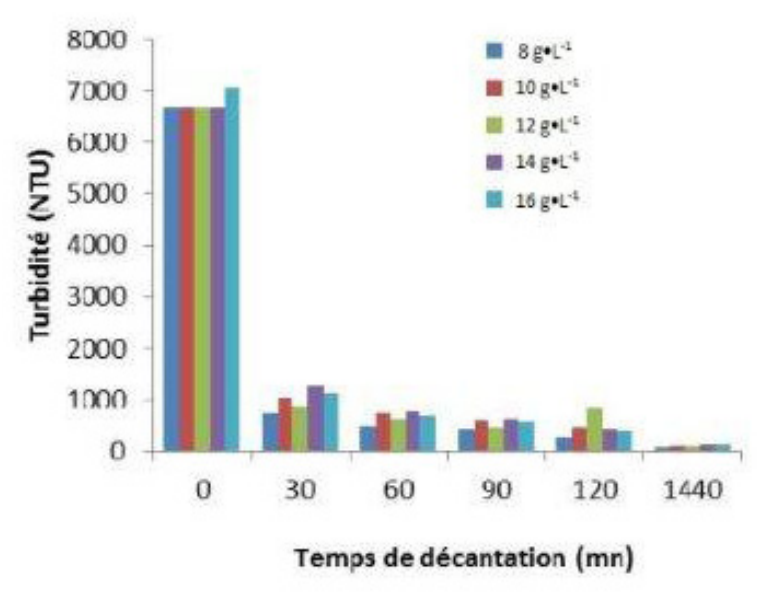

(c)

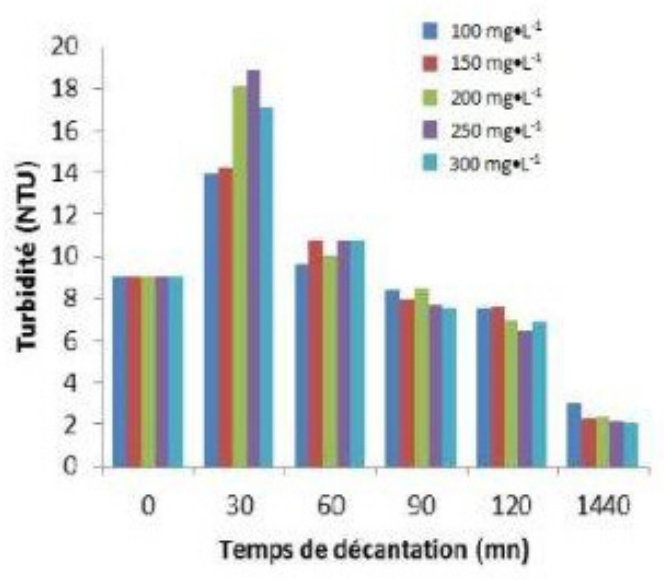

(b)

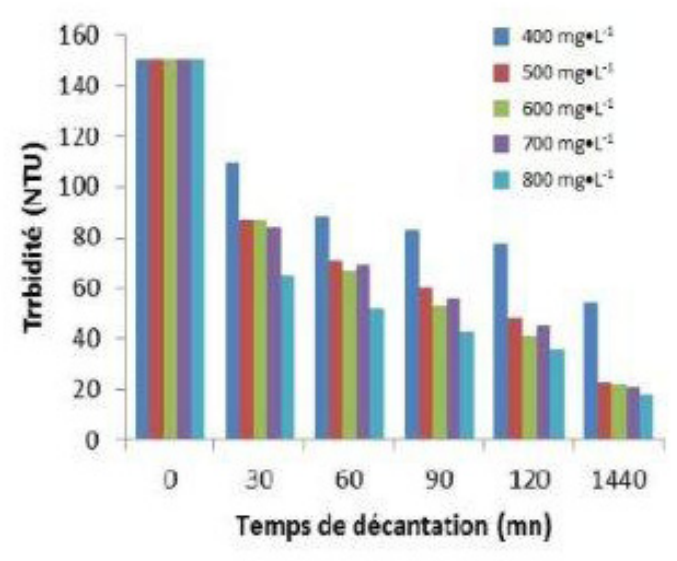

(d)

Figure 1. Évolution de la turbidité des eaux en fonction du temps de décantation pour différentes concentrations de Moringa oleifera : (a) Loumbila, (b) Ouaga3, (c) Gana, (d) Bouaré.

Evolution of the water sample turbidity in relation to the settling time for different concentrations of Moringa oleifera: (a) Loumbila, (b) Ouaga3, (c) Gana, (d) Bouaré. 
sur ces types d'eau lorsque l'on utilise les graines de $M$. oleifera (FOLKARD, 1997; JAHN, 1988a). Ainsi, FOLKARD (1997) a proposé que pour ces types d'eaux (turbidité inférieure à 35 NTU), la méthode de contact floculation-filtration qui consiste à introduire le coagulant dans l'eau brute juste avant l'entrée dans un filtre est l'idéale. Pour cette méthode, la floculation et le dépôt des flocs se font directement dans le filtre, rendant le traitement plus efficace.

Le traitement de l'échantillon de Bouaré (eau de puits contenant de fines particules d'argile) a aussi nécessité des concentrations assez élevées $\left(400-800 \mathrm{mg}^{\bullet} \mathrm{L}^{-1}\right)$ au regard de la turbidité initiale (150 NTU). Pour cette eau, l'abattement de la turbidité obtenu pour un temps de décantation de deux heures et 24 heures et pour une concentration de $800 \mathrm{mg} \bullet \mathrm{L}^{-1}$ de $M$. oleifera a été de $75,34 \%$ et $87,58 \%$ pour des valeurs respectives de turbidité de 36,7 et 18,5 NTU. Ces valeurs de turbidité sont nettement supérieures à celle recommandée par l'OMS ( $\leq 5 \mathrm{NTU}$ ). La formation de très petits flocs observée pour cette eau entraîne une clarification lente et imparfaite (Figure 1d). Cela s'explique par les faibles poids moléculaires des particules (argiles) qui sédimentent très lentement.

Concernant l'échantillon du Gana qui présentait une turbidité élevée (6 $681 \mathrm{NTU}$ ), de fortes concentrations de coagulant $\left(>7 \mathrm{~g} \bullet \mathrm{L}^{-1}\right)$ ont été utilisées. Un abattement rapide de la turbidité au bout de 30 minutes de décantation a été observé pour toutes les concentrations de $M$. oleifera. La concentration de $8 \mathrm{~g} \bullet \mathrm{L}^{-1}$ de $M$. oleifera donne la meilleure turbidité aussi bien pour deux heures (262 NTU) et 24 heures (74 NTU) de décantation pour cette eau pour des abattements respectifs de $96,07 \%$ et $98,88 \%$. Au-delà de cette concentration, la turbidité de l'eau reste uniformément élevée due à un excès de matière organique du coagulant (Figure 1c). Toutefois, bien que la clarification soit très importante, le traitement demeure inefficace pour obtenir de l'eau propre à la consommation même au bout de 24 heures. Ainsi, les échantillons fortement chargés en matières organiques (turbidité > 1000 NTU) nécessitent des doses de $M$. oleifera très élevées (FOLKARD, 1997).

En ce qui concerne l'échantillon d'eau de Loumbila, la concentration de $250 \mathrm{mg}^{\bullet} \mathrm{L}^{-1}$ de $M$. oleifera donne la meilleure valeur de turbidité aussi bien pour deux heures (6 NTU) que 24 heures de décantation (4 NTU) pour des abattements de $90,18 \%$ et $93,7 \%$ respectivement au bout de deux et 24 heures de décantation. À cette concentration, la turbidité obtenue après 24 heures de décantation $(4 \mathrm{NTU})$ repond à la norme fixée par l'OMS (Figure 1a).

Les graines de $M$. oleifera contiennent un polypeptide basique, plus précisément un ensemble de poly-électrolytes cationiques actifs de 12-14 kDa (KATRE et al., 2008;
NDABIGENGESERE et al.,1995; OKUDA et al.,1999). Ces poly-électrolytes de charge positive neutralisent les colloïdes des eaux troubles car la majorité de ces colloïdes ont une charge négative (FOILD et al., 2002). De l'ensemble des résultats, il ressort que l'efficacité du traitement des eaux avec les graines de $M$. oleifera varie d'une eau à une autre. Les doses requises dans le traitement des eaux au $M$. oleifera varient en fonction du taux de matière organique présent dans l'eau, de la turbidité initiale de celle-ci et de la nature des éléments à floculer, notamment celle des argiles (FABY et ELELI, 1993; FOLKARD, 1997).

Les temps de décantation compris entre 1,5 et 2 heures ont permis une élimination très significative de la turbidité des eaux. Bien que des temps de décantation de 24 heures donnent les meilleures valeurs de turbidité, la qualité organoleptique des eaux est fortement affectée (odeur, goût, aspect) du fait de la présence de matière organique des graines de $M$. oleifera. En accord avec nos résultats, JAHN (1988a) a conseillé une décantation de 1-2 heures, suivie d'une filtration et chloration, afin d'éliminer les particules et les microorganismes résiduels en suspension.

Selon FOLKARD et al. (2002), la dose de graines de Moringa oleifera nécessaire pour le traitement se situe entre 75 et $200 \mathrm{mg} \bullet \mathrm{L}^{-1}$, selon la turbidité initiale de l'eau. Nos études ont montré que pour des eaux moyennement turbides (75-100 NTU), la dose optimale de coagulant se situe entre 250 et $300 \cdot \mathrm{L}^{-1}$ pour obtenir des valeurs répondant à la norme de potabilité des eaux de boisson (échantillons de Loumbila). Pour des eaux contenant des particules d'argiles, les concentrations nécessaires sont également élevées du fait du faible poids moléculaire des particules qui sédimentent très lentement. C'est le cas de l'échantillon de Bouaré pour lequel une concentration de $800 \mathrm{mg} \cdot \mathrm{L}^{-1}$ a été utilisée pour ramener la turbidité de 150 à 18,5 NTU. Ces résultats montrent qu'en fonction du degré de pollution des eaux les concentrations de coagulant varient. Toutefois FOLKARD (1997), FOLKARD et SUTHERLAND (1992) ont montré que l'abattement de la turbidité n'est pas proportionnel à la quantité de $M$. oleifera mais tient compte aussi de la qualité de la graine (l'origine de l'arbre), c'est-à-dire de la concentration en protéines actives.

\subsection{Influence du traitement sur le $p H$ des échantillons d'eaux}

Les $\mathrm{pH}$ des eaux utilisées pour cette étude sont proches de la neutralité (entre 7 et 8). Les variations de $\mathrm{pH}$ enregistrées avant et après traitement sont comprises respectivement entre 0,15-1,73 unité $\mathrm{pH}$. Ainsi, la composition chimique $(\mathrm{pH}$, conductivité) des eaux évolue peu après traitement au M. oleifera (FOLKARD, 1997). Cela est en accord avec nos résultats qui indiquent que le traitement influe peu sur le $\mathrm{pH}$ de l'eau dont la variation n'est pas statistiquement significative 
sauf pour les échantillons du Gana où la dose utilisée est très élevée.

\subsection{Effet du traitement sur les paramètres physico-chimiques des eaux}

Le tableau 2 présente les résultats de l'analyse de variance des concentrations de nitrates, nitrites, calcium, magnésium, matières organiques, matières en suspension et la dureté totale des eaux déterminées avant et aux conditions optimales de traitement.

Il en ressort que le traitement a un effet très significatif sur tous les paramètres ciblés $(\mathrm{p}<0,0001)$. Une baisse très significative des concentrations de nitrates, calcium, magnésium et dureté totale a été observée pour tous les échantillons, contre une augmentation de celles des sulfates, matières organiques et matières en suspension pour tous les échantillons excepté celui du Gana.

Le tableau 3 présente l'évolution des paramètres physicochimiques des eaux avant et après traitement.

Pour les échantillons de Loumbila et de Ouaga3 qui contenaient de faibles concentrations de nitrates $\left(1 \mathrm{mg} \bullet \mathrm{L}^{-1}\right)$, le traitement $\mathrm{a}$ induit une augmentation de près de $100 \%$ qui pourrait être due à la présence de nitrates dans le coagulant (cf. Tableau 1). Ce léger apport en nitrates n'affecte nullement la qualité de ces eaux dont le seuil de tolérance est de $50 \mathrm{mg} \bullet \mathrm{L}^{-1}$ (WHO, 2011).

Pour les échantillons de Gana et Bouaré, par contre, les concentrations en nitrates ont significativement baissé respectivement de 98 et $60 \%$ aux conditions optimales de traitement. La coagulation avec des graines de $M$. oleifera est basée sur l'adsorption et la neutralisation dans l'eau de particules chargées négativement (colloïdes) et de métaux par des charges positives des protéines actives (VIKASHNI et al., 2012). Ce mécanisme pourrait expliquer l'élimination des nitrates, nitrites ainsi que du calcium, magnésium et de la dureté totale avec les colloïdes pour ces échantillons $(\mathrm{p}<0,0001)$. Pour tous les échantillons, une augmentation des sulfates (50 à $150 \%$ ), des matières organiques (30 à $100 \%$ ) et des matières en suspension (35 à $48 \%$ ) a été observée avec le traitement excepté celui du Gana pour lequel une baisse des matières organiques et des matières en suspension a été observée. Malgré l'apport d'ions sulfates dans l'eau traitée par le coagulant, les concentrations finales dans les eaux traitées répondent à la norme OMS dont le seuil est fixé à $250 \mathrm{mg} \bullet \mathrm{L}^{-1}$. Du fait du taux élevé de matières organiques dans le coagulant, le traitement a induit une augmentation de la concentration de matières organiques dans les eaux traitées. En accord avec nos résultats, FATOMBI et al. (2009) ont montré que les graines de Moringa oleifera

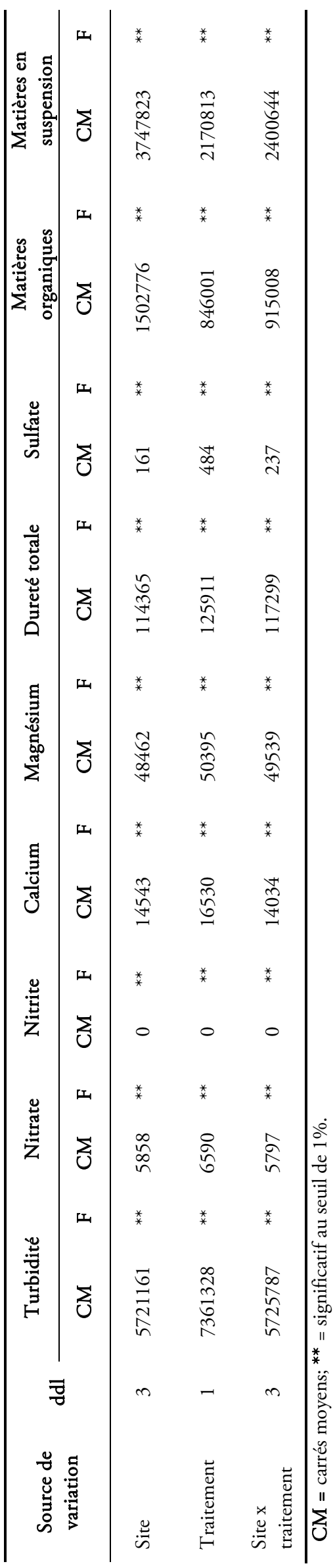


contiennent près de $94 \%$ de matières organiques et entraînent une augmentation du taux de matières organiques dans l'eau traitée (entre 100 à $400 \%$ ), et également de la DBO et DCO.

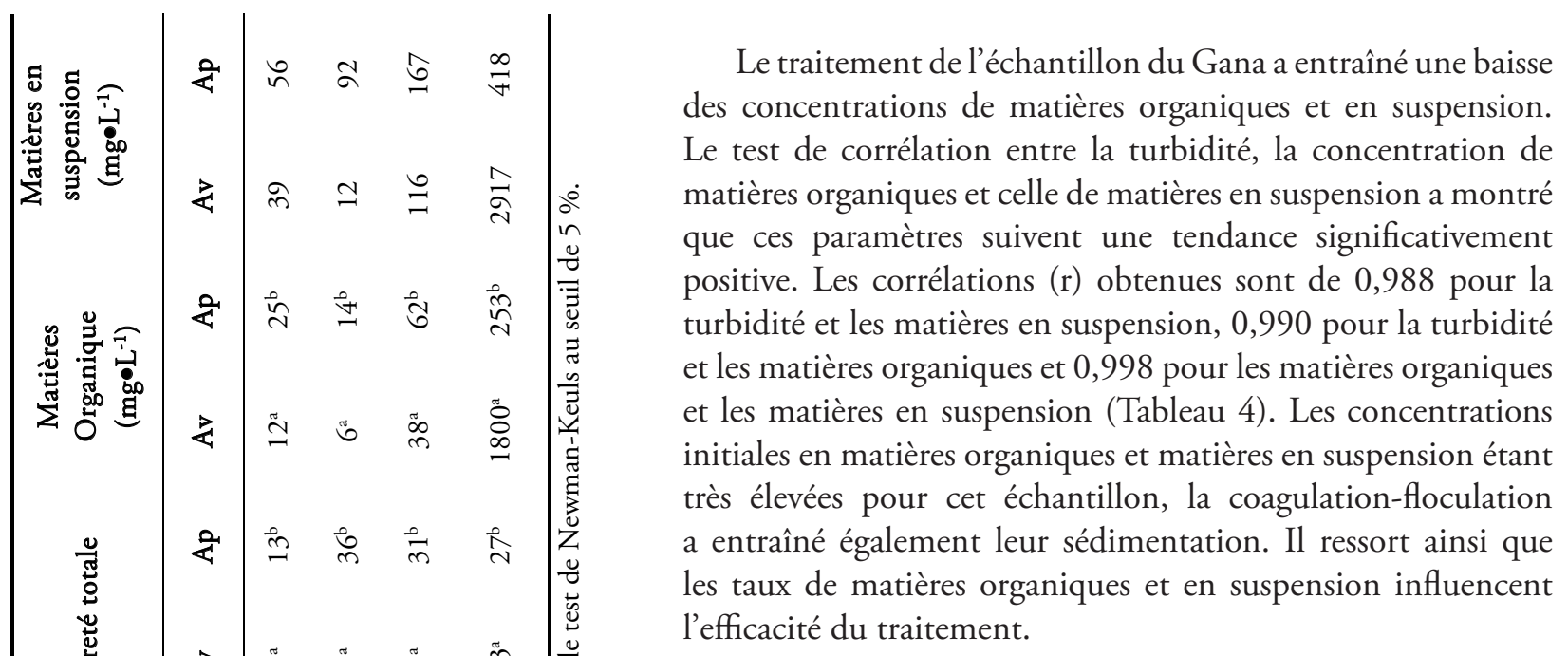

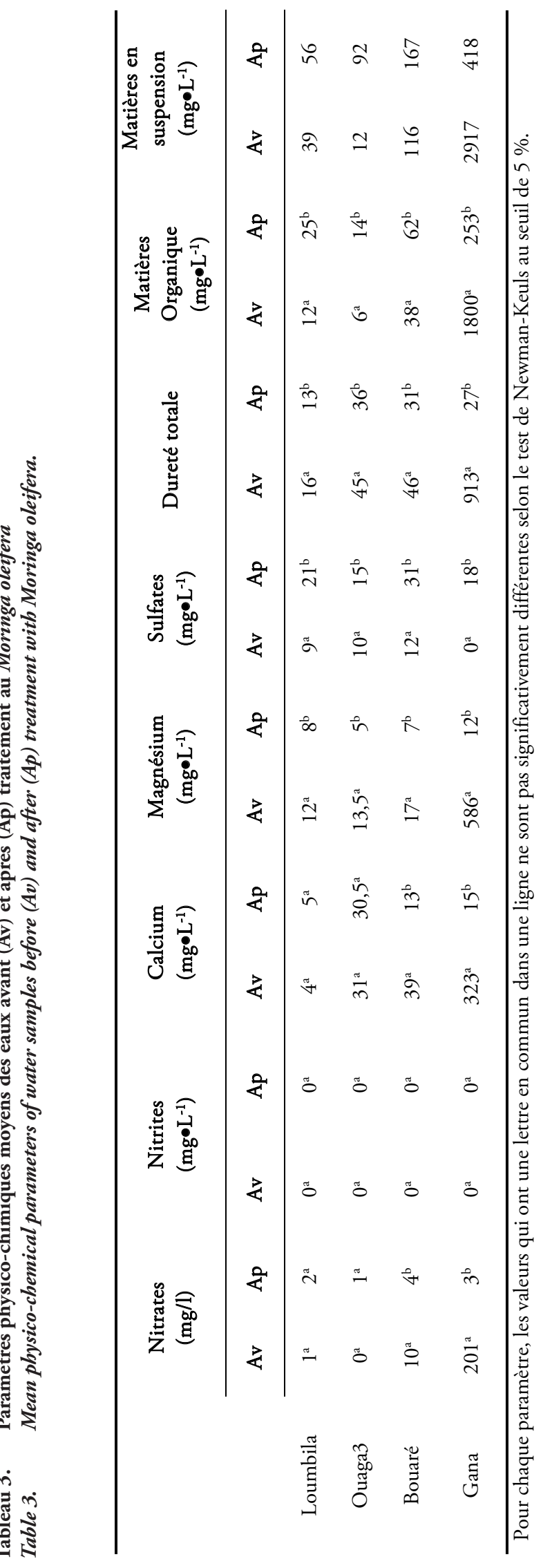

\subsection{Evolution des indicateurs microbiens dans les échantillons d'eau en fonction du traitement au $M$. oleifera}

Les teneurs en indicateurs microbiens de contamination fécale des eaux avant et après traitement au $M$. oleifera sont présentées par la figure 2 .

L'analyse microbiologique des surnageants des échantillons d'eau traités a montré une élimination très importante de tous les germes au bout de deux heures de décantation. Pour les échantillons d'eau de Loumbila et Ouaga3 (Figures 2a, 2b), l'abattement des coliformes fécaux est statistiquement significatif au seuil $5 \%$ pour toutes les concentrations de $M$. oleifera testées $(\mathrm{p}<0,0001)$. Les concentrations de $250 \mathrm{mg} \bullet \mathrm{L}^{-1}$ et $300 \mathrm{mg} \bullet \mathrm{L}^{-1}$ conduisent également à une élimination totale d'E. coli et des streptocoques fécaux respectivement pour ces deux échantillons. $250 \mathrm{mg} \cdot \mathrm{L}^{-1}$ de $M$. oleifera a permis un abattement de $100 \%$ des streptocoques fécaux et d'E. coli et $82 \%$ des coliformes fécaux. Par contre, pour l'échantillon de Ouaga3, la concentration de $300 \mathrm{mg} \bullet \mathrm{L}^{-1}$ de coagulant a permis un abattement de $100 \%$ d'E. coli et $75 \%$ des coliformes fécaux. Aucun kyste de parasites n'a été retrouvé dans les échantillons d'eau de ces deux sites.

Quant aux échantillons d'eau de Bouaré et du Gana, bien que des réductions significatives $(\mathrm{p}<0,0001)$ des teneurs en coliformes fécaux, $E$. coli et streptocoques fécaux soient obtenues aux concentrations de $M$. oleifera donnant les meilleures clarifications ( 800 et $8000 \mathrm{mg}^{\bullet} \mathrm{L}^{-1}$ respectivement), les teneurs finales de ces indicateurs de pollution fécale restent toutefois supérieures aux seuils recommandés par l'OMS (2011) pour l'eau de boisson (Figures 2c, 2d). Pour l'échantillon de Bouaré (Figure 2c) les abattements ont été de $74 \%$ pour les coliformes fécaux, $87 \%$ pour $E$. coli et près de 
Tableau 4. Corrélation entre turbidité, matière organique et matière en suspension.

Table 4. Correlation between turbidity, suspended matter and organic matter.

\begin{tabular}{ccc}
\hline Sources de corrélation & $\mathbf{r}$ & $\mathrm{p}$ \\
\hline Turbidité $\times$ MES & 0,988 & $<0,0001$ \\
Turbidité $\times$ MO & 0,990 & $<0,0001$ \\
$\mathrm{MO} \times \mathrm{MES}$ & 0,998 & $<0,0001$ \\
\hline
\end{tabular}

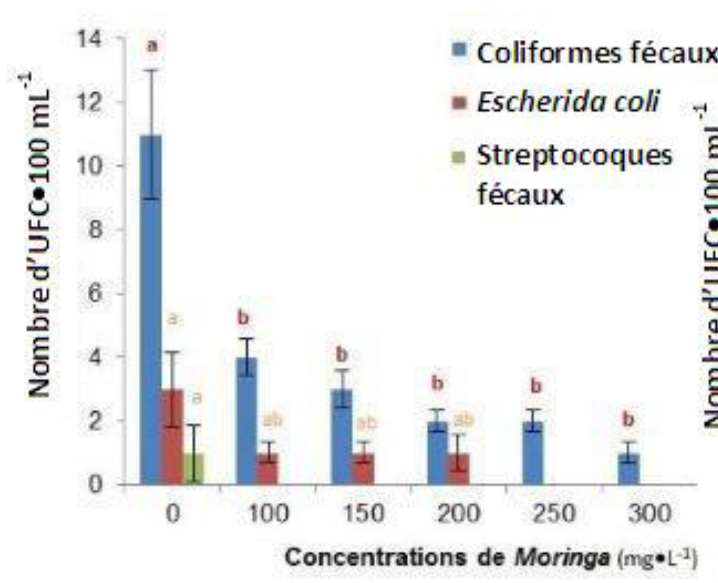

(a)

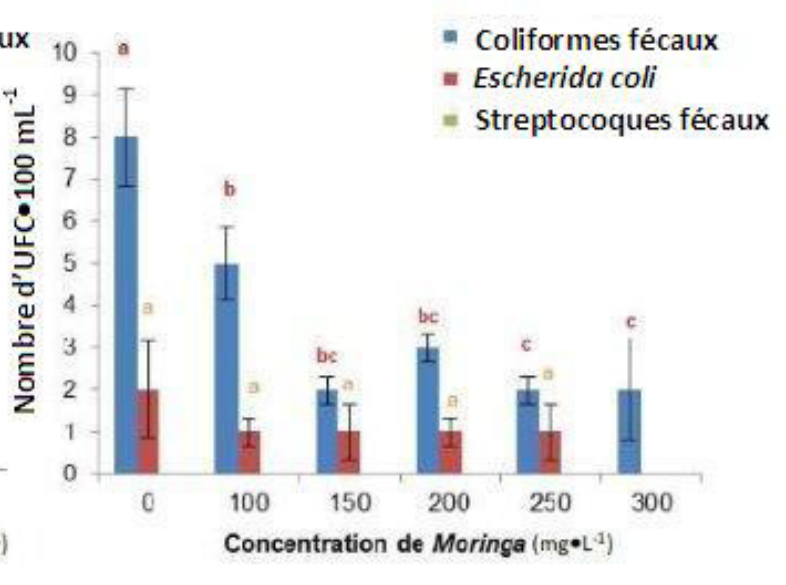

(b)

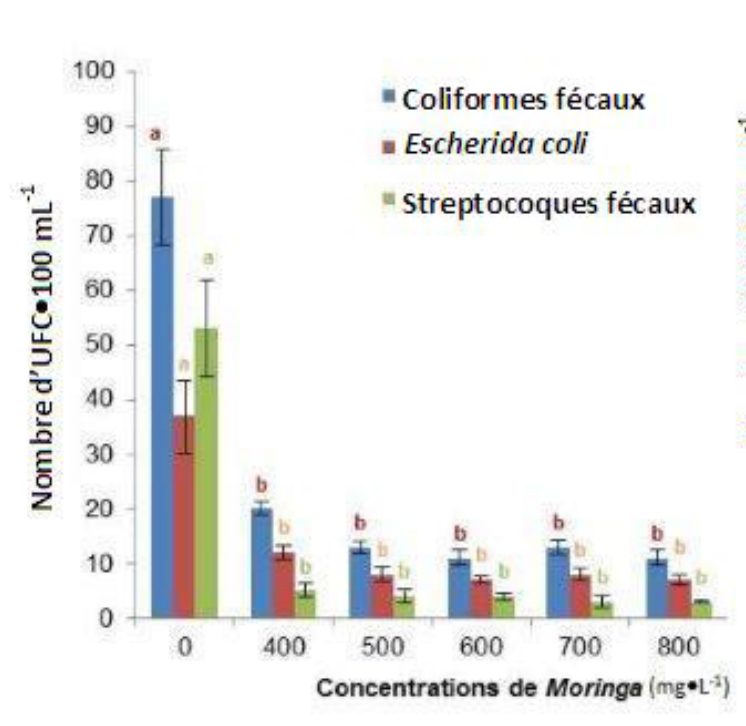

(c)

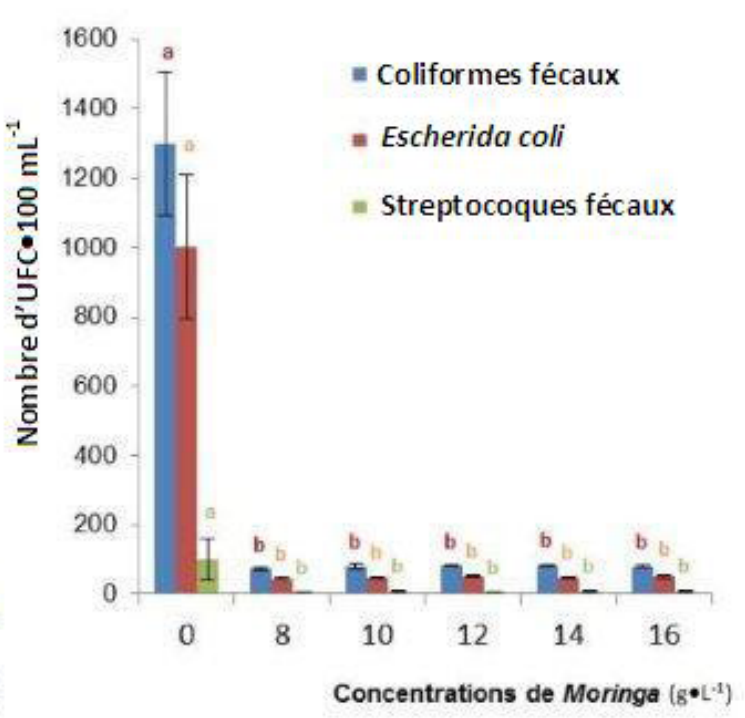

(d)

Figure 2. Évolution des indicateurs microbiens de contamination fécale dans les échantillons eaux en fonction des concentrations Moringa oleifera : (a) Loumbila, (b) Ouaga3, (c) Bouaré; (d) Gana. Evolution of microbial indicators of fecal contamination in water samples in relation to the concentration of Moringa oleifera: (a) Loumbila, (b) Ouaga3, (c) Bouaré, (d) Gana. 
$90 \%$ pour les streptocoques fécaux. Aucun kyste de parasites n'a été retrouvé dans cet échantillon. Le traitement s'est révélé moins performant surtout pour l'échantillon du Gana très pollué au plan bactériologique. Les abattements obtenus pour cet échantillon ont été de l'ordre de $95 \%$ pour les coliformes fécaux, $96 \%$ pour les $E$. coli et $94 \%$ pour les streptocoques fécaux. Quant aux kystes de parasites, principalement ceux de Giardia intestinalis détectés dans les échantillons de ce site, l'abattement a été de $100 \%$. Sur un plan d'ensemble, les eaux de ces deux sites demeurent impropres à la consommation humaine.

L'efficacité du traitement sur les microorganismes est fonction du degré de pollution initiale des eaux. Aussi, les abattements microbiens sont fonction des caractéristiques physico-chimiques et microbiologiques initiales de l'eau brute et proportionnels à l'abattement de la turbidité (BRATBY, 2006). En effet, étant donné que les microorganismes sont rattachés aux particules en suspension, leur sédimentation entraîne également celle des microorganismes. Ces facteurs expliquent ainsi les différences observées au niveau des abattements obtenus. Ainsi, plus la clarification est parfaite et la qualité microbiologique initiale acceptable, plus la qualité microbiologique de l'eau traitée est meilleure. Le traitement au $M$. oleifera est suffisant si l'eau à traiter est de bonne qualité bactériologique (JAHN, 1989). Ces résultats sont en accord avec ceux obtenus par FOLKARD et SUTHERLAND (1992) puis FABY et ELELI (1993). Selon JAHN (1989), l'inconvénient majeur est l'apport de matière organique apportée par les graines qui peut favoriser, à long terme, la croissance bactérienne dans l'eau traitée, d'où la nécessité d'un temps de stockage relativement court (pas plus de 24 heures). En effet, l'abondance des bactéries dépend de la quantité de substances nutritives présentes dans l'eau sous forme de carbone organique (sucre, acides aminés, acides organiques, etc.). Une eau potable ne doit contenir que 0,5 à $2 \mathrm{mg}^{\bullet} \mathrm{L}^{-1}$ de carbone organique dissout afin de garantir sa stabilité du point de vue microbiologique (LAUTENSCHLAGER K. et al., 2010). Ainsi, du fait de la présence de matières organiques dans l'eau traitée, le temps de stockage doit être réduit pour éviter une recontamination de l'eau traitée à travers la prolifération de microorganismes.

Sur un plan d'ensemble, les résultats de cette étude font ressortir que lorsque l'eau brute n'est pas trop chargée en microorganismes, le traitement par coagulation-floculation avec les graines de $M$. oleifera peut être suffisant pour avoir de l'eau potable. Par contre, lorsqu'elle a une turbidité élevée et est trop chargée en microorganismes, le traitement par coagulation-floculation apparaît insuffisant pour avoir de l'eau potable et les eaux doivent subir un traitement additionnel avant toute consommation.

\section{CONCLUSION ET PERSPECTIVES}

L'eau potable est un enjeu sanitaire majeur pour les populations, en particulier dans les pays en développement. Les méthodes simples et peu coûteuses de traitement des eaux sont un critère essentiel de la durabilité des processus dans ces pays. Selon JAHN (1988b), les plantes utilisées pour la floculation doivent remplir certaines conditions. Elles doivent être faciles à produire et leur coagulant facile à doser. En outre, elles ne doivent pas présenter de toxicité. Les graines de $M$. oleifera répondent très bien à ces critères. Les plantes utilisées doivent aussi être cultivables dans de nombreux pays sans nécessiter trop d'espace. $M$. oleifera est particulièrement facile à cultiver de manière intensive et est adapté à la plupart des zones tropicales. Bien que l'efficacité des graines soit fonction de la qualité initiale de l'eau brute, le traitement permet une nette amélioration de la qualité des eaux de consommation.

À partir de ces résultats prometteurs il sera intéressant d'étendre la recherche sur des méthodes de réduction de la matière organique résiduelle dans l'eau traitée afin de stabiliser l'eau traitée et d'étendre la durée de conservation.

\section{REMERCIEMENTS}

Cette recherche a été réalisée grâce au soutien financier de ISP/Suède, UEMOA et de la Fondation Internationale pour la Science, Suède, par l'intermédiaire d'une Bourse de recherche $\left(\mathrm{N}^{\circ} \mathrm{W} / 5405-1\right)$ à qui les auteurs adressent leurs remerciements.

\section{RÉFÉRENCES BIBLIOGRAPHIQUES}

\author{
AOUBA S. (2012). Indicateurs empiriques du développement \\ humain durable: cas de l'accès à l'eau potable dans la commune \\ rurale de Saaba au Burkina Faso. Mémoire de Maîtrise en \\ Macroéconomie et Gestion de Développement, USTA, \\ Ouagadougou, Burkina Faso, 50 p.
}

BUISSON Y. (2001). Les diarrhées, un problème de santé publique. Conférence inaugurale Institut Pasteur du Cambodge, Phnom Penh, Cambodge. Med. Trop., 61, 205-209.

BRATBY J. (2006). Coagulation and flocculation in water and wastewater treatment. Seconde édition, IWA Publishing, Londres, $450 \mathrm{p}$. 
DEGREMONT (2005). Mémento technique de l'eau : Tome 2. Lavoisier SAS (Éditeur) - Lexique technique de l'eau, Paris, $10^{\mathrm{e}}$ édition, $785 \mathrm{p}$.

DESJARDINS R. (1997). Le traitement des eaux. Presses Internationales Polytechniques, Éditions de l'École Polytechnique de Montréal, Canada. Deuxième édition revue et enrichie, $540 \mathrm{p}$.

DIANOU D., J.N. PODA, L.G. SAVADOGO, H. SORGHO, S.P. WANGO et B. SONDO (2004). Parasitoses intestinales dans la zone du complexe hydroagricole du Sourou au Burkina Faso. Vertigo, 5, 1-8.

DIANOU D., J.N. PODA, H. SORGHO, S.P. WANGO et K.B. SONDO (2003). Hydraulic planning and schistosomiasis: Case of Sourou in Burkina Faso. J. Appl. Res. Vet. Med., 1, 105-111.

DIANOUD., B. SAVADOGO, D.ZONGO, T.ZOUGOURI, J.N. PODA, H. BADO et F. ROSILLON (2011). Qualité des eaux de surface dans la vallée du Sourou : cas des rivières Mouhoun, Sourou, Débé et Gana au Burkina Faso. Int. J. Biol. Chem. Sci., 5, 1571-1589.

FABY J.A. et A. ELELI (1993). Utilisation de la graine de Moringa, essais de floculation au laboratoire et en vraie grandeur. CIEH/EIER/Oieau, Série hydraulique urbaine et assainissement, Ouagadougou, Burkina Faso, 132 p.

FATOMBI K.J., R.G JOSSE., D. MAMA et T. AMINOU (2009). Étude de l'activité floculante de la caséine acide extraite de la crème de Cocos nucifera dans la clarification des eaux de surface. Rev. Sci. Eau, 22, 93-101.

FOILD N., H.P.S. MAKKAR et K. BECKER (2002). Potentiel du Moringa oleifera pour les besoins agricoles et industriels. Dans : Potentiel de Développement Pour Les Produits de Moringa. Acte de l'Atelier International de Dar es Salam Saint-Sauveur, Appora, Besse et Fuglie, 29 octobre-2 novembre 2001, Tanzanie, (Cd-Rom) MONTPELLIER (Éditeur).

FOLKARD G. (1997). The development of the Moringa oleifera and stenopetala tree to provide valuable products: coagulant for water/wastewater treatment and vegetable oil. Rapport à la Commission Européenne, DG 12, projet de recherche No TS3CT94-0309, période 1995-1997.

FOLKARD G. et J. SUTHERLAND (1992). Development of robust water treatment systems incorporating natural coagulants. Field study report, January-March 1992, Thyolo, Malawi $16 \mathrm{p}$.
FOLKARD G. et J. SUTHERLAND (2002). Development of a naturally derived coagulant for water and wastewater treatment. Water Suppl., 2, 89-94.

HOSSAM A. et A. ALGHAMDI (2011). Enhancement of quality of secondary industrial wastewater effluent by coagulation process: A case Study. J. Environ. Protec., 2, 1250-1256.

JAHN S.A.A. (1989). Différents rôles des coagulants naturels dans la clarification de l'eau, dans les technologies appropriées à usage domestique et dans les installations communales d'épuration. Dans : Proceedings of International Seminar on the Use of Natural Coagulants for Water Treatment, Yogyakarta, Indonesia, 2-7 octobre, 11 p.

JAHN S.A.A. (1988a). Using Moringa seeds as coagulants in developing countries. J. AWWA, 80, 43-50.

JAHN S.A.A. (1988b). Chemotaxonomy of flocculating plant materials and their application for rural water purification in developing countries. Acta Univ. Ups. Symb. Bot. Ups., $28,171-185$.

KABORE A. (2011). Étude du pouvoir floculant et des qualités épuratoires des graines de Moringa oleifera dans le traitement des eaux brutes de consommation en Afrique sub-saharienne: Cas des eaux du Burkina Faso. Mémoire de DEA, Université de Ouagadougou, Ouagadougou Burkina Faso, 54 p.

KATRE U.V., C.G. SURESH, M.I. KHAN et S.M. GAIKWAD (2008). Structure-activity relationship of a hemagglutinin from Moringa oleifera seeds. Int. J. Biol. Macromol., 42, 203-207.

KOUKOUNARI A., A.F. GABRIELLI, S. TOURE, E. BOSQUE-OLIVA, Y. ZHANG, B. SELLIN, C.A. DONNELLY, A. FENWICK et J.P. WEBSTER (2007). Schistosoma haematobium infection and morbidity before and after large-scale administration of Praziquantel in Burkina Faso. J. Infect. Dis., 196, 659-669.

LAUTENSCHLAGER K., N. BOON, Y. WANG, T. EGLI et F. HAMMES (2010). Overnight stagnation of drinking water in household taps induces microbial growth and changes in community composition. Water Res., 44. 4868-4877.

MAHRH (Ministère de l'Agriculture, de l'Hydraulique et des Ressources Halieutiques) (2011). Rapport bilan GIRE Burkina Faso (2010) et perspectives 2011. Ouagadougou, Burkina Faso, 29 p. 
NDABIGENGESERE A., K.S. NARASIAH et B.G. TALBOT (1995). Active agents and mechanism of coagulation of turbid waters using Moringa oleifera. Water Res., 29, 703-710.

OKUDA T., A.U. BAES, W. NISHIJIMA et M. OKADA (1999). Improvement of extraction method of coagulation active components from Moringa oleifera seed. Water Res., 33, 3373-3378.

OMS (2005). Les effets de l'environnement sur la santé de la mère et de l'enfant. OMS, fév. 2005, aide-mémoire $\mathrm{N}^{\circ} 284$, Genève, Suisse.

OMS/UNICEF (2000). Global water supply and sanitation assessment report, Genève, Suisse, $77 \mathrm{p}$.

PN-AEPA (2006). Programme national d'approvisionnement en eau potable et d'assainissement à l'horizon 2015. Ministère de l'Agriculture, de l'Hydraulique et des Ressources Halieutiques, Direction Générale des Ressources en Eau PN-AEPA, Burkina Faso, 55 p.

ROSILLON F., B. SAVADOGO, A. KABORE, H. BABOSAMA et D. DIANOU (2012b). Estimation of the nitrates contents in waters by using reagent strips: An environment education exercise in the Sourou Valley in Burkina Faso. Vertigo, 12, publié en ligne, septembre 2012 (http:// vertigo.revues.org/12274).

TRAORE I. (2003). Impact des facteurs géographiques sur le développement des Schistosomiases dans la vallée du Sourou. Mémoire de Maitrise en Géographie, Université de Ouagadougou, Burkina Faso, 119 p.

UNITED NATIONS, UNDP, Burkina Faso (2010). Access to safe drinking water has improved a lot. http://www.un.org/ apps/newsFr/printnewsAr.asp?nid=23248, (consulté le 09/01/2013).

VIKASHNI N., M. MATAKITE, K. KANAYATHU et S. SUBRAMANIUM (2012). Water purification using Moringa oleifera and other locally available seeds in Fiji for heavy metal removal. Int. J. Appl. Sci. Technol., 2, 125-129.

WHO (2011). Guidelines for drinking water quality (4 $4^{e}$ édition). WHO, ISBN 978924154815 1, Genève, Suisse, 531 p. 